\title{
Assessing the impact of coordinated COVID-19 exit strategies across Europe
}

\author{
N. W. Ruktanonchai1 ${ }^{12 * \dagger}$, J. R. Floyd ${ }^{1 * \dagger}$, S. Lai ${ }^{1 * \dagger}$, C. W. Ruktanonchai ${ }^{1}$, A. Sadilek ${ }^{3}$, P. Rente-Lourenco ${ }^{4}$, \\ X. Ben ${ }^{3}$, A. Carioli' , J. Gwinn ${ }^{5}$, J. E. Steele ${ }^{1}$, O. Prosper6 ${ }^{6}$ A. Schneider ${ }^{3}$, A. Oplinger ${ }^{3}$, P. Eastham $^{3}$, A. J. Tatem $^{1}$ \\ ${ }^{1}$ WorldPop, School of Geography and Environmental Science, University of Southampton, Southampton, UK. ${ }^{2}$ Population Health Sciences, Virginia Tech, Blacksburg, VA, \\ USA. ${ }^{3}$ Google, Mountain View, CA, USA. ${ }^{4}$ Vodafone Group, London, UK. ${ }^{5}$ College of Public Health, University of Kentucky, Lexington, KY, USA. ${ }^{6}$ Department of Mathematics, \\ University of Tennessee, Knoxville, TN, USA. \\ *Corresponding author. Email: nrukt00@gmail.com (N.W.R.); jrf1g15@soton.ac.uk (J.R.F.); shengjie.lai@soton.ac.uk (S.L.)
}

†These authors contributed equally to this work.

As rates of new COVID-19 cases decline across Europe due to non-pharmaceutical interventions such as social distancing policies and lockdown measures, countries require guidance on how to ease restrictions while minimizing the risk of resurgent outbreaks. Here, we use mobility and case data to quantify how coordinated exit strategies could delay continental resurgence and limit community transmission of COVID-19. We find that a resurgent continental epidemic could occur as many as 5 weeks earlier when wellconnected countries with stringent existing interventions end their interventions prematurely. Further, we found that appropriate coordination can greatly improve the likelihood of eliminating community transmission throughout Europe. In particular, synchronizing intermittent lockdowns across Europe meant half as many lockdown periods were required to end community transmission continent-wide.

The ongoing COVID-19 pandemic rapidly spread across Europe throughout February and March 2020, making it the largest cluster of cases worldwide (1). In response, most of Europe implemented strict lockdown measures to control disease spread, which have been shown to be effective at reducing transmission (2-4). As rates of new cases decline, countries are now implementing various exit strategies to relax restrictions (5). Long-term success of any potential exit strategy hinges on what happens regionally, as international importation could overwhelm efforts to prevent resurgence through testing and contact tracing $(6,7)$. To account for this, the European Commission recommended that governments provide advance warning of plans to relax non-pharmaceutical interventions (NPIs) (8), and in particular, has focused on coordinated easing of travel restrictions (9). To better inform the importance and nature of an internationally coordinated exit strategy, governments require an evidence base for understanding importation and the consequences of easing interventions in an uncoordinated way.

Data from mobile phones can help address this by informing connectivity patterns, contact rates, and the effect of various NPIs on mobility. In other settings, they have been instrumental for understanding where infection occurs for various diseases (10) such as malaria $(11,12)$, predicting disease spread (13), and quantifying population mobility during and after catastrophic events (14). More recently, for the COVID-19 pandemic, mobile phone data have been valuable in assessing NPI effectiveness $(3,4)$, and remain at the forefront of understanding whether populations are adhering to social distancing policies (15-18). These data link well with theoretical models that provide a basis for understanding how heterogeneous mobility and exposure will affect disease invasion (19) in spatially structured populations (20), as well.

Here we provide an evidence base for coordinated exit strategies across Europe using mobile phone data and a metapopulation model of COVID-19 transmission (21). Specifically, we quantify the progression of a second epidemic continent-wide if countries act in a coordinated or uncoordinated manner. We also quantify how coordination could influence regionally interrupted transmission of COVID-19, testing the importance of synchronized NPIs if countries phase them to limit economic impact. We accomplished this by (i) estimating pre-COVID-19 mobility using a novel anonymized and aggregated call data record (CDR) dataset from Vodafone and an anonymized and aggregated continental NUTS3 (Nomenclature of Territorial Units for Statistics) mobility dataset from Google (table S1), (ii) measuring mobility reductions due to NPIs using a separate COVID-19 Google dataset, and (iii) propagating these reductions in an epidemiological model (see fig. S1 for data flow). All analyses were undertaken at the NUTS3 administrative unit level, which are administrative boundaries regulated by the European Union (EU) for use within EU member states (22), with spatial extents defined by population thresholds ranging between 150,000 to 800,000 residents. 


\section{Baseline mobility and COVID-19-related reductions}

First, we predicted the baseline probability of moving between NUTS3 regions across Europe using the Vodafone data in Spain and Italy and the continental Google NUTS3 dataset (Fig. 1).

We then analyzed the Google COVID-19 dataset to quantify reductions in mobility and contact rates from January 2020 through the end of March 2020 in response to the COVID-19 pandemic (Fig. 2). In our simulations, we used observed reductions in mobility in each NUTS3 area to proportionally reduce outgoing flows, incoming flows, and local contact rates for that area.

Using these baseline mobility patterns and reductions in mobility, we simulated the spread of COVID-19 over 6 months starting 4 April 2020 while making various assumptions about where and when NPIs would be relaxed or reinstated. Across all simulations, we started transmission on 20 March 2020 because this predates large reductions in mobility (Fig. 2 , right), allowing the disease to spread initially in a datadriven way that can help account for spatial biases in reporting and testing. We parameterized initial numbers of people infected using a repository maintained by the Johns Hopkins University CSSE (23). Because the case data from this repository were country-level, we distributed cases across NUTS3 area proportionally based on population size (fig. S10).

To simulate different exit strategies and the overall impact of different NPIs enacted, we reduced mobility one week past 28 March (for 29 March-4 April) based on the observed change between 15-21 March to 22-28 March to account for changes caused by further uptake of existing NPIs (Fig. 2). On average, this represented an overall mean $65 \%$ reduction in mobility compared to 28 January-18 February, agreeing with recent studies on contact rate reductions in the UK (24), which observed a $73 \%$ reduction in daily contacts. When simulating active lockdowns on dates past 4 April, we used the predicted mobility reduction for each NUTS3 area from 29 March-4 April. When we simulated countries lifting their NPIs entirely, we used the relative mobility patterns observed during 1-7 March.

\section{Modeling the effect of lifting interventions early}

First, we compared secondary epidemic timing when all countries coordinated their exit strategies with simulations where one country ended their interventions early. We iteratively tested the impact of each country in Europe easing lockdowns starting 15 April, while all other countries extended their NPIs for 4, 8, and 12 weeks, depending on simulation run. For the country that lifted their NPIs early, we assumed people in each NUTS3 area would voluntarily reduce their average contact rate by $20 \%$ compared to the January-February baseline, or slightly less than the reduction in mobility observed on 23 March, because countries that have lifted NPIs have observed sustained limited mobility reductions beyond the relaxing of various restrictions (16).

If a country lifted their NPIs early, we found a second epidemic could occur much earlier (Fig. 3, left). The right panel of Fig. 3 measures the earlier timing to $25 \%$ of people across Europe having had COVID-19 (infected + recovered + exposed; see fig. S13 for plot showing this explicitly). This measure captures when uncontrolled widespread transmission occurred while accounting for multiple peaks and varying peak heights in Fig. 3. Time to $25 \%$ infected was particularly sensitive to well-connected countries that implemented strong NPIs, such as France and Italy (Fig. 3, right). France lifting their NPIs early led to the earliest second epidemic, 35 days earlier than if all countries lifted their NPIs simultaneously (interquartile range from 32.3 to 36.8 days). Despite having experienced relatively low reductions in mobility through 28 March, Germany remains important to continental resurgence, due to high connectivity with neighboring countries (Fig. 1, right). When exploring the epidemic curves through time when different countries lifted their NPIs early, we found that different types of mobility initiated continental epidemics. While France lifting their NPIs early led to resurgence in major population centers continent-wide, Germany lifting NPIs early led to resurgence in neighboring countries first (fig. S15). Further, certain areas keeping $R$ slightly over 1 under NPIs also led to an initial peak in some simulations, and maintained the threat of resurgence even after 12 weeks of NPIs continent-wide (Fig. 3, left; see supplementary materials section "Exploring spatiotemporal dynamics of spread" for more detail). This occurred in central Turkey in our simulations and dramatically affected continental spread. In simulations where smaller or less-connected countries lifted their NPIs early, we found that resurgence was largely driven by importation from central Turkey and exhibited epidemic curves very similar to the case where all countries maintained NPIs (Fig. 3, red line).

\section{Modeling the effect of synchronized intermittent NPIs}

We also tested how cycling NPIs in a synchronized or unsynchronized way affected the continent-wide epidemic. Cycled NPIs meant countries switched between being under interventions for several weeks and under no interventions for the same number of weeks over several cycles. Synchronized NPIs meant all countries implemented lockdowns at the same time, while unsynchronized NPIs meant half of all countries (randomly chosen for each simulation run) were under lockdown at any time. Cycling NPIs reflects the intermittent lockdowns that could occur if countries reinstate interventions after surpassing threshold numbers of new cases $(25,26)$. Therefore, this test helps predict what may happen if countries do not coordinate the easing and reinstating of NPIs based on regional rates of new cases. Here, we ran 
various simulations where lockdown and non-lockdown periods were 3 or 4 weeks long over 2, 3, or 4 cycles in total.

Across 1200 simulations, we found that synchronized cycles of NPIs were always more likely to end community transmission over 6 months, and generally lowered transmission further than if NPIs were unsynchronized (Fig. 4). In the most striking example, synchronizing four cycles of three week long lockdowns led to local elimination COVID-19 cases in $90 \%$ of simulations, while unsynchronized cycles only led to elimination $5 \%$ of the time (Fig. 4 , bottom left). Two synchronized cycles of four-week NPIs were also sufficient to end community transmission, whereas four unsynchronized cycles of four-week NPIs were necessary to end community transmission (Fig. 4, right). The only simulations where unsynchronized NPIs had fewer cases than synchronized NPIs at the end of simulation was with 2 cycles of 3 week-long NPIs (Fig. 4, top left), which occurred because enough people were infected under unsynchronized NPIs that herd immunity reduced transmission. Importantly, these simulations do not include any importation from other regions of the globe. As a result, simulations reflecting zero local cases after certain numbers of intermittent lockdowns are very unlikely to be realized. Instead, this result reflects the likelihood of reducing local cases to a low enough level that strong test-and-trace systems can catch importations before significant outbreaks occur.

Intergovernmental organizations such as the World Health Organization have stressed the importance of international solidarity in terms of sharing resources and expertise in combating COVID-19 (1). Our results reiterate this, as one country ending NPIs before others could mean disease resurgence across Europe as many as 5 weeks earlier, reducing the time available to expand test-and-treat and to develop new therapeutics or vaccines (Fig. 3). Heterogeneities in mobility reduction (Fig. 2), baseline mobility patterns (Fig. 1), and population sizes mean that certain countries are particularly important to continental resurgence, such as France, Germany, Italy, and Poland (Fig. 3).

These key countries varied in how local cases led to continental resurgence, implying different key interventions for each. For example, while spread out of Germany led to epidemics in neighboring countries initially, spread out of France led to epidemics in population centers continent-wide (fig. S12). Further, we found that small pockets of community transmission under NPIs could assure a second epidemic wave continent-wide. In our study, this occurred due to central Turkey experiencing limited mobility reductions (Fig. 2) and exhibiting a high starting reproductive number (fig. S2) that kept local $R$ slightly above 1 when under NPIs. While the actual mobility reduction in central Turkey is uncertain and has likely changed since late March 2020, this highlights the importance of countries ensuring that $R$ stays below 1 during lockdown periods, and the importance of effective screening of international travelers from areas with sustained transmission well into the future.

We also found that the nature of coordination was key to reducing resurgence risk. When cycling NPIs between weeks, synchronized interventions across all countries meant that cases could be driven down more quickly (Fig. 4). Fewer cases at the end of the synchronized lockdowns led to much higher likelihoods of reaching zero cases locally, due to a higher chance of stochastic recovery processes leading to interrupted transmission. In real terms, the synchronized scenario approximates what could happen if countries set case thresholds for lifting NPIs regionally, while the unsynchronized scenario simulates what could happen if countries only consider case numbers within their boundaries.

This study has several limitations that influence the direct applicability of our case number predictions across Europe. We also use observed mobility reductions as a proxy for reductions in contact rate, which may not reflect reality, though the contact rate reduction estimates from this process accord with those observed in other studies (24). Secondly, COVID19 is known to exhibit age-dependent severity, and contact rates are strongly age-dependent $(27,28)$, which could introduce heterogeneities that we did not incorporate into our simulations. We believe our results should be robust to these limitations, however, because we ran our simulations over different values of $R$ and varying the serial interval. In these sensitivity analyses, we found that the existence of key countries and the importance of coordinated NPIs were robust to these changes (see supplementary materials), though varying the serial interval could extend or shrink the second epidemic timings and epidemic peaks observed in Fig. 3. Additionally, we reduced mobility for each NUTS3 area uniformly, but long-distance movement reduced much more than short-distance movement due to country-level travel restrictions and other NPIs (fig. S8). This likely provides a continental protective effect against early resurgence compared to our results, particularly if quarantining measures are put in place for long-distance travelers.

Our mobility estimates may also be biased due to the populations included in the Google and Vodafone data. Google's consumer Location History feature is only available from smartphone users, is turned off by default, and is viewed through the lens of differential privacy algorithms designed to protect user privacy and obscure fine detail. Vodafone's anonymized and aggregated data were based on network data from customers who had full control over their privacy settings, potentially introducing biases as well. This work makes a step toward using multiple datasets to capture population-level patterns that go beyond any one service or system. Further, because both the Google and Vodafone data are aggregate datasets, we could not account for individual-level 
correlation in mobility patterns in our model (i.e., individuals who travel elsewhere but return home shortly thereafter). This likely means our model will overestimate spread and resurgence in general, as infectious people will end up less likely to return home.

Coordination will be key to an effective, equitable response to COVID-19. This means not just sharing resources, but also ensuring that exit strategies account for neighboring countries and regions. While coordinating exit strategies across an entire continent may prove politically difficult, the presence of key countries and community structure offer possible coordination groups that do not require engagement from all countries. We have explored some of these coordination groups in a community detection analysis in the supplementary materials (fig. S16). Further, coordinated exit strategies that account for real-time case data will likely improve outcomes compared to our predictions, as we simulated intermittent NPIs that were lifted regardless of actual transmission context. A multifaceted, reactive approach to lifting NPIs will be necessary to minimize resurgence risk. This means beyond international cooperation, robust testand-treat (29) and household quarantine (30) measures should be in place. Future work will further inform the role mobility, NPIs, and international coordination can play in slowing COVID-19 resurgence, building on existing work (31) examining invasion, re-invasion, and disease extinction (32) in spatially structured populations. Critically, even if community transmission is reduced to very low levels within Europe (for example, through intermittent NPIs shown in Fig. 4), importation from other regions of the globe mean coordination will be necessary to prevent epidemics continent-wide well into the future.

The implications of our study extend well beyond Europe and COVID-19, broadly demonstrating the importance of communities coordinating easing of various NPIs for any potential pandemic. In the United States, NPIs have been generally implemented at the state-level, and because states will be strongly interconnected, our results emphasize national coordination of pandemic preparedness efforts moving forward. Elsewhere, relatively porous national borders between many lower and middle income countries mean without coordination, these countries may have to deal with significant international importation after controlling local transmission (33). COVID-19 transmission and transmission of any infectious disease will ignore national and provincial borders; preventing resurgence and spread will mean ensuring that pockets of transmission do not persist in areas with limited interventions at the expense of later epidemics in others.

\section{REFERENCES AND NOTES}

1. C. Sohrabi, Z. Alsafi, N. O'Neill, M. Khan, A. Kerwan, A. Al-Jabir, C. Iosifidis, R. Agha, World Health Organization declares global emergency: A review of the 2019 novel coronavirus (COVID-19). Int. J. Surg. 76, 71-76 (2020). doi:10.1016/j.ijsu.2020.02.034 Medline
2. K. Prem, Y. Liu, T. W. Russell, A. J. Kucharski, R. M. Eggo, N. Davies, M. Jit, P. Klepac, S. Flasche, S. Clifford, C. A. B. Pearson, J. D. Munday, S. Abbott, H. Gibbs, A. Rosello, B. J. Quilty, T. Jombart, F. Sun, C. Diamond, A. Gimma, K. van Zandvoort, S. Funk, C. I. Jarvis, W. J. Edmunds, N. I. Bosse, J. Hellewell; Centre for the Mathematical Modelling of Infectious Diseases COVID-19 Working Group, The effect of control strategies to reduce social mixing on outcomes of the COVID-19 epidemic in Wuhan, China: A modelling study. Lancet Public Health 5, e261-e270 (2020). doi:10.1016/S2468-2667(20)30073-6 Medline

3. S. Lai, N. W. Ruktanonchai, L. Zhou, O. Prosper, W. Luo, J. R. Floyd, A. Wesolowski, M. Santillana, C. Zhang, X. Du, H. Yu, A. J. Tatem, Effect of non-pharmaceutical interventions to contain COVID-19 in China. Nature (2020). doi:10.1038/s41586020-2293-x Medline

4. M. U. G. Kraemer, C.-H. Yang, B. Gutierrez, C.-H. Wu, B. Klein, D. M. Pigott, L. du Plessis, N. R. Faria, R. Li, W. P. Hanage, J. S. Brownstein, M. Layan, A. Vespignani, H. Tian, C. Dye, O. G. Pybus, S. V. Scarpino; Open COVID-19 Data Working Group, The effect of human mobility and control measures on the COVID-19 epidemic in China. Science 368, 493-497 (2020). doi:10.1126/science.abb4218 Medline

5. T. Colbourn, COVID-19: Extending or relaxing distancing control measures. Lancet Public Health 5, e236-e237 (2020). doi:10.1016/S2468-2667(20)30072-4 Medline

6. S. Lai, I. Bogoch, N. Ruktanonchai, A. Watts, X. Lu, W. Yang, H. Yu, K. Khan, A. J. Tatem, Assessing spread risk of Wuhan novel coronavirus within and beyond China, January-April 2020: a travel network-based modelling study. medRxiv 2020.02.04.20020479 [Preprint]. 9020. https://doi.org/10.1101/2020.02.04.20020479

7. M. Chinazzi, J. T. Davis, M. Ajelli, C. Gioannini, M. Litvinova, S. Merler, A. Pastore Y Piontti, K. Mu, L. Rossi, K. Sun, C. Viboud, X. Xiong, H. Yu, M. E. Halloran, I. M. Longini Jr., A. Vespignani, The effect of travel restrictions on the spread of the 2019 novel coronavirus (COVID-19) outbreak. Science 368, 395-400 (2020). Medline

8. M. McKee, A European roadmap out of the covid-19 pandemic. BMJ 369, m1556 (2020). doi:10.1136/bmi.m1556 Medline

9. U. von der Leyen, C. Michel, "Joint European Roadmap towards lifting COVID-19 containment measures" (Communication, European Commission, Brussels, Belgium, 2020); https://ec.europa.eu/info/sites/info/files/communication a_european_roadmap_to_lifting coronavirus containment_measures_0.pdf.

10. A. Wesolowski, C. O. Buckee, K. Engø-Monsen, C. J. E. Metcalf, Connecting mobility to infectious diseases: The promise and limits of mobile phone data. J. Infect. Dis. 214 (suppl. 4), S414-S420 (2016). doi:10.1093/infdis/jiw273 Medline

11. N. W. Ruktanonchai, P. DeLeenheer, A. J. Tatem, V. A. Alegana, T. T. Caughlin, E. Zu Erbach-Schoenberg, C. Lourenço, C. W. Ruktanonchai, D. L. Smith, Identifying malaria transmission foci for elimination using human mobility data. PLOS Comput. Biol. 12, e1004846 (2016). doi:10.1371/journal.pcbi.1004846 Medline

12. D. K. Pindolia, A. J. Garcia, A. Wesolowski, D. L. Smith, C. O. Buckee, A. M. Noor, R. W. Snow, A. J. Tatem, Human movement data for malaria control and elimination strategic planning. Malar. J. 11, 205 (2012). doi:10.1186/1475-2875-11-205 Medline

13. A. J. Tatem, D. L. Smith, International population movements and regional Plasmodium falciparum malaria elimination strategies. Proc. Natl. Acad. Sci. U.S.A. 107, 12222-12227 (2010). doi:10.1073/pnas.1002971107 Medline

14. R. Wilson, E. Zu Erbach-Schoenberg, M. Albert, D. Power, S. Tudge, M. Gonzalez, S. Guthrie, H. Chamberlain, C. Brooks, C. Hughes, L. Pitonakova, C. Buckee, X. Lu, E. Wetter, A. Tatem, L. Bengtsson, Rapid and near real-time assessments of population displacement using mobile phone data following disasters: The 2015 Nepal earthquake. PLOS Curr. 8 10.1371/currents.dis.d073fbece328e4c39087bc086d694b5c doi:10.1371/currents. dis.d073fbece328e4c39087bc086d694b5c Medline

15. L. Queiroz, A. Ferraz, J. L. Melo, G. Barboza, A. Urbanski, A. Nicolau, S. Oliva, H. Nakaya, "Large-scale assessment of human mobility during COVID-19 outbreak," Open Science Framework Preprints (2020); https://doi.org/10.31219/osf.io/nqxrd.

16. Google LLC, "COVID-19 Community Mobility Reports"; www.google.com/covid19/mobility.

17. N. M. Kavanagh, R. R. Goel, A. S. Venkataramani, Association of county-level socioeconomic and political characteristics with engagement in social distancing 
for COVID-19. medRxiv 2020.04.06.20055632 [Preprint]. 11 April 2020. https://doi.org/10.1101/2020.04.06.20055632.

18. A. L. Wright, K. Sonin, J. Driscoll, J. Wilson, "Poverty and Economic Dislocation Reduce Compliance with COVID-19 Shelter-in-Place Protocols" (University of Chicago, Becker Friedman Institute for Economics Working Paper No. 2020-40, 2020); http://dx.doi.org/10.2139/ssrn.3573637.

19. P. C. Cross, J. O. Lloyd-Smith, P. L. F. Johnson, W. M. Getz, Duelling timescales of host movement and disease recovery determine invasion of disease in structured populations. Ecol. Lett. 8, 587-595 (2005). doi:10.1111/j.1461$0248.2005 .00760 . x$

20. V. Colizza, A. Vespignani, Epidemic modeling in metapopulation systems with heterogeneous coupling pattern: Theory and simulations. J. Theor. Biol. 251, $450-$ 467 (2008). doi:10.1016/i.jtbi.2007.11.028 Medline

21. N. Ruktanonchai, wpgp/BEARmod: EU model code, Zenodo (2020); https://doi.org/10.5281/zenodo.3932111.

22. R. Smith, in Core EU Legislation (Macmillan Education UK, 2015), pp. 183-186.

23. Johns Hopkins University Center for Systems Science and Engineering, 2019 Novel Coronavirus COVID-19 (2019-nCoV) Data Repository by Johns Hopkins CSSE (2020); https://github.com/CSSEGISandData/COVID-19.

24. C. I. Jarvis, K. van Zandvoort, A. Gimma, K. Prem, CMMID nCov Working Group, P. Klepac, G. J. Rubin, W. J. Edmunds, "Impact of physical distance measures on transmission in the UK," Centre for Mathematical Modelling of Infectious Diseases Repository (2020); https://cmmid.github.io/topics/covid19/comix-impact-ofphysical-distance-measures-on-transmission-in-the-UK.html.

25. L. Tarrataca, C. M. Dias, D. B. Haddad, E. F. Arruda, Flattening the curves: on-off lock-down strategies for COVID-19 with an application to Brazil. arXiv:2004.06916 [q-bio.PE] (15 April 2020).

26. M. Gollwitzer, C. Platzer, C. Zwarg, A. S. Göritz, Public acceptance of potential Covid-19 lockdown scenarios. PsyArXiv (14 April 2020); https://doi.org/10.31234/osf.io/3a85z.

27. G. Onder, G. Rezza, S. Brusaferro, Case-fatality rate and characteristics of patients dying in relation to COVID-19 in Italy. JAMA 323, 1775-1776 (2020). doi:10.1001/jama.2020.4683 Medline

28. F. Zhou, T. Yu, R. Du, G. Fan, Y. Liu, Z. Liu, J. Xiang, Y. Wang, B. Song, X. Gu, L. Guan, Y. Wei, H. Li, X. Wu, J. Xu, S. Tu, Y. Zhang, H. Chen, B. Cao, Clinical course and risk factors for mortality of adult inpatients with COVID-19 in Wuhan, China: A retrospective cohort study. Lancet 395, 1054-1062 (2020). doi:10.1016/S01406736(20)30566-3 Medline

29. Royal Society DELVE Initiative, "Test, Trace, Isolate" (27 May 2020); https://rsdelve.github.io/reports/2020/05/27/test-trace-isolate.html.

30. A. Aleta, D. Martin-Corral, A. Pastore y Piontti, M. Ajelli, M. Litvinova, M. Chinazzi, N. E. Dean, M. E. Halloran, I. M. Longini, S. Merler, A. Pentland, A. Vespignani, E. Moro, Y. Moreno, Modeling the impact of social distancing, testing, contact tracing and household quarantine on second-wave scenarios of the COVID-19 epidemic. medRxiv 2020.05.06.20092841 [Preprint]. 18 May 2020. https://doi.org/10.1101/2020.05.06.20092841.

31. C. Poletto, M. Tizzoni, V. Colizza, Human mobility and time spent at destination: Impact on spatial epidemic spreading. J. Theor. Biol. 338, 41-58 (2013). doi:10.1016/i.jtbi.2013.08.032 Medline

32. G.-Q. Sun, Q.-X. Liu, Z. Jin, A. Chakraborty, B.-L. Li, Influence of infection rate and migration on extinction of disease in spatial epidemics. J. Theor. Biol. 264, 95-103 (2010). doi:10.1016/i.jtbi.2010.01.006 Medline

33. K. Wangdi, M. L. Gatton, G. C. Kelly, A. C. A. Clements, in Advances in Parasitology, Volume 89, D. Rollinson, J. R. Stothard, Eds. (Academic Press, 2015), pp. 79-107.

34. European Commission, Eurostat, Statistics Explained, Glossary:Country codes; https:/ec.europa.eu/eurostat/statisticsexplained/index.php/Glossary:Country codes.

35. N. Ruktanonchai, Synthetic data based on IPUMS microcensus data for mobility between NUTS3 areas of Europe, Version 1, Zenodo (2020); https://doi.org/10.5281/zenodo.3931987.

36. N. W. Ruktanonchai, C. W. Ruktanonchai, J. R. Floyd, A. J. Tatem, Using Google Location History data to quantify fine-scale human mobility. Int. J. Health Geogr. 17, 28 (2018). doi:10.1186/s12942-018-0150-z Medline

37. L. Forsberg White, M. Pagano, A likelihood-based method for real-time estimation of the serial interval and reproductive number of an epidemic. Stat. Med. 27,
2999-3016 (2008). doi:10.1002/sim.3136 Medline

38. I. Mulheirn, S. Alvis, L. Insall, J. Browne, C. Palmou, "A sustainable exit strategy: Managing uncertainty, minimising harm" (Tony Blair Institute for Global Change, 2020); https://institute.global/policy/sustainable-exit-strategy-managinguncertainty-minimising-harm

39. European Centre for Disease Prevention and Control, Download today's data on the geographic distribution of COVID-19 cases worldwide (2020); www.ecdc.europa.eu/en/publications-data/download-todays-data-geographicdistribution-covid-19-cases-worldwide.

40. Q. Li, X. Guan, P. Wu, X. Wang, L. Zhou, Y. Tong, R. Ren, K. S. M. Leung, E. H. Y. Lau, J. Y. Wong, X. Xing, N. Xiang, Y. Wu, C. Li, Q. Chen, D. Li, T. Liu, J. Zhao, M. Liu, W. Tu, C. Chen, L. Jin, R. Yang, Q. Wang, S. Zhou, R. Wang, H. Liu, Y. Luo, Y. Liu, G. Shao, H. Li, Z. Tao, Y. Yang, Z. Deng, B. Liu, Z. Ma, Y. Zhang, G. Shi, T. T. Y. Lam, J. T. Wu, G. F. Gao, B. J. Cowling, B. Yang, G. M. Leung, Z. Feng, Early transmission dynamics in Wuhan, China, of novel coronavirus-infected pneumonia. N. Engl. J. Med. 382, 1199-1207 (2020). doi:10.1056/NEJMoa2001316 Medline

41. S. Abbott, J. Hellewell, R. Thompson, K. Sherratt, H. P. Gibbs, N. I. Bossee, J. D. Munday, S. Meakin, E. L. Doughty, J. Y. Chun, Y.-W. D. Chan, F. Finger, P. Campbell, A. Endo, C. A. B. Pearson, A. Gimma, T. Russell, CMMID COVID modelling group, S. Flasche, A. J. Kucharski, R. M. Eggo, S. Funk, "Methods: Summary, introduction, data sources, accounting for delays, statistical methods, summary of results and limitations"; https://epiforecasts.io/covid/methods.html.

42. Assessment Capacities Project (ACAPS), \#COVID19 Government Measures Dataset (2020); www.acaps.org/covid19-government-measures-dataset.

43. N. G. Davies, A. J. Kucharski, R. M. Eggo, A. Gimma, W. J. Edmunds, T. Jombart, K. O'Reilly, A. Endo, J. Hellewell, E. S. Nightingale, B. J. Quilty, C. I. Jarvis, T. W. Russell, P. Klepac, N. I. Bosse, S. Funk, S. Abbott, G. F. Medley, H. Gibbs, C. A. B. Pearson, S. Flasche, M. Jit, S. Clifford, K. Prem, C. Diamond, J. Emery, A. K. Deol, S. R. Procter, K. van Zandvoort, Y. F. Sun, J. D. Munday, A. Rosello, M. Auzenbergs, G. Knight, R. M. G. J. Houben, Y. Liu; Centre for the Mathematical Modelling of Infectious Diseases COVID-19 working group, Effects of non-pharmaceutical interventions on COVID-19 cases, deaths, and demand for hospital services in the UK: A modelling study. Lancet 5, e375-e385 (2020). doi:10.1016/S24682667(20)30133-X Medline

44. Newzoo, Top Countries by Smartphone Users; https://newzoo.com/insights/rankings/top-countries-by-smartphonepenetration-and-users/.

45. A. Bassolas, H. Barbosa-Filho, B. Dickinson, X. Dotiwalla, P. Eastham, R. Gallotti, G. Ghoshal, B. Gipson, S. A. Hazarie, H. Kautz, O. Kucuktunc, A. Lieber, A. Sadilek, J. J. Ramasco, Hierarchical organization of urban mobility and its connection with city livability. Nat. Commun. 10, 4817 (2019). doi:10.1038/s41467-019-12809-y Medline

46. R. J. Wilson, C. Y. Zhang, W. Lam, D. Desfontaines, D. Simmons-Marengo, B. Gipson, Differentially Private SQL with Bounded User Contribution. arXiv:1909.01917 [cs.CR] (25 November 2019).

\section{ACKNOWLEDGMENTS}

The authors would like to acknowledge JP, SW, EF and the DG group for insightful discussions on heterogeneously applied interventions. Funding: This study was supported by the grants from the Vodafone Institute; the Bill \& Melinda Gates Foundation (OPP1134076); the European Union Horizon 2020 (MO0D 874850). AJT is supported by funding from the Bill \& Melinda Gates Foundation (INV002697, OPP1106427, OPP1032350, OPP1134076, OPP1094793), the Clinton Health Access Initiative, the UK Department for International Development (DFID) and the Wellcome Trust (106866/Z/15/Z, 204613/Z/16/Z). NR is supported by funding from the Bill \& Melinda Gates Foundation (OPP1170969, OPP1209812). OP is supported by the National Science Foundation (1816075). Author contributions: NWR, JRF, SL, PRL, AJT designed the research. NWR, JRF, SL, CWR, PRL, OP, AC built the model and ran simulations. NWR, JRF, SL, CWR, AS, PRL, AC, carried out analyses. AS, PRL, XB, AC, JES, AS, AO, PE provided technical support. AS, PRL, XB, AS, AO, PE helped with data curation. AS and XB created the Google COVID-19 aggregated dataset. NWR, JRF, SL, CWR, AC, AS, PRL, JG, OP, AJT wrote and edited the manuscript. Competing interests: Authors declare no competing interests. Data and materials availability: Code for the model simulations is available at the following GitHub 
repository: https://github.com/wpgp/BEARmod. The specific version of this model used in this study, with simulation code, R estimates shown in fig. S2, and initial numbers infected shown in fig. S10 can be found at

https://doi.org/10.5281/zenodo.3932111 (21). The population movement data obtained from Google and Vodafone for this study are not publicly available due to stringent licensing agreements. The Google COVID-19 Aggregated Mobility Research Dataset used for this study is available with permission from Google LLC (contact: Adam Sadilek, sadilekadam@google.com). The Vodafone datasets can also be accessed with the permission of Vodafone through correspondence with the corresponding authors. A synthetic NUTS3-level dataset of mobility across Europe is also available at https://doi.org/10.5281/zenodo.3931987

(35). This work is licensed under a Creative Commons Attribution 4.0

International (CC BY 4.0) license, which permits unrestricted use, distribution, and reproduction in any medium, provided the original work is properly cited. To view a copy of this license, visit

https://creativecommons.org/licenses/by/4.0/. This license does not apply to figures/photos/artwork or other content included in the article that is credited to a third party; obtain authorization from the rights holder before using such material.

\section{SUPPLEMENTARY MATERIALS}

science.sciencemag.org/cgi/content/full/science.abc5096/DC1

Materials and Methods

Supplementary Text

Figs. S1 to S16

Tables S1 and S2

References (36-46)

MDAR Reproducibility Checklist

1 May 2020; accepted 13 July 2020

Published online 17 July 2020

10.1126/science.abc5096 


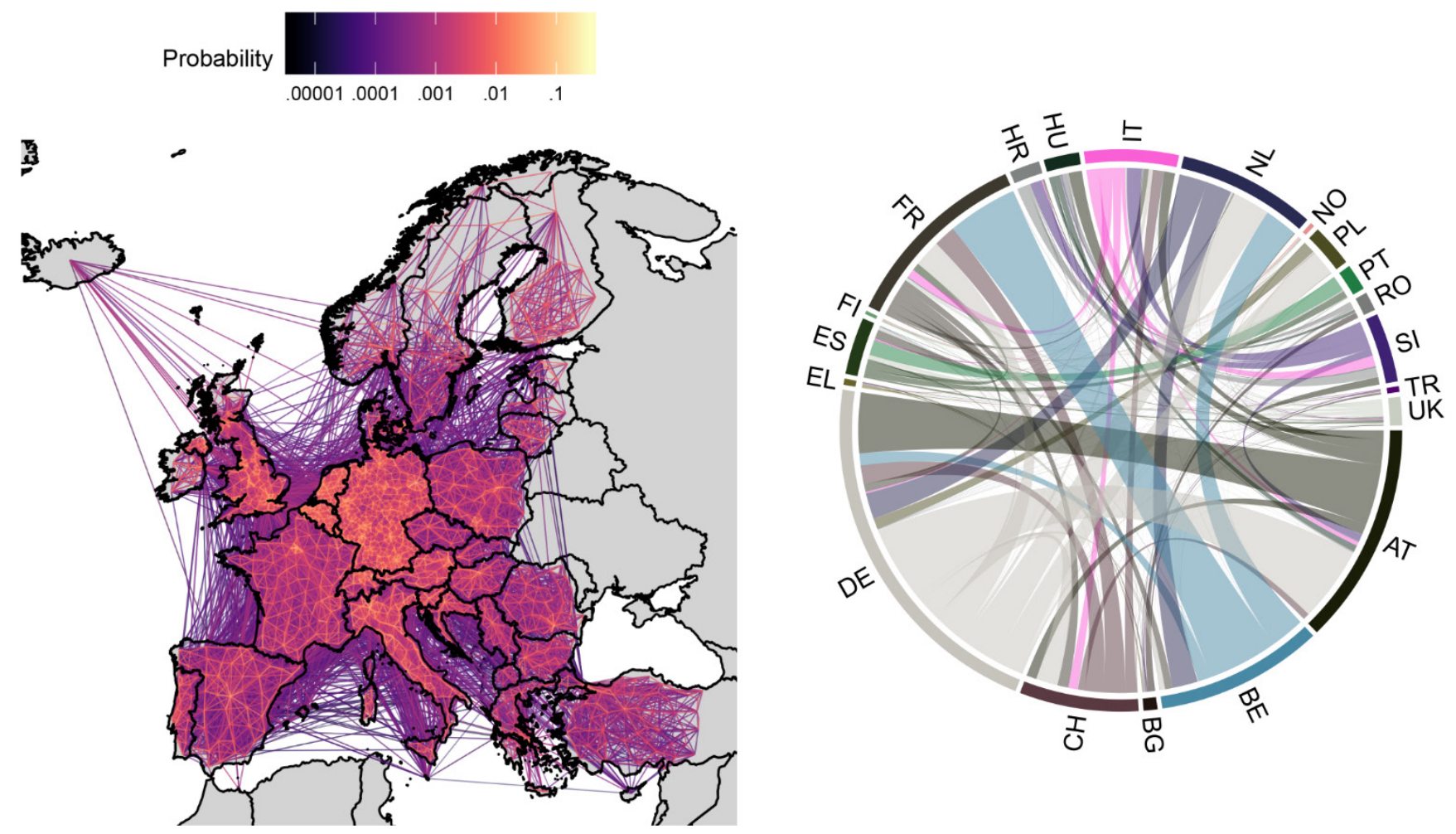

Fig. 1. Predicted baseline mobility patterns for 28 Jan-18 Feb 2020. (Left) Probability of people moving between NUTS3 administrative units per 8 hours. (Right) Individual probability of moving between top 20 European countries with the greatest outward mobility. For example, an individual in Germany (DE) is roughly twice as likely to travel internationally as compared to an individual in Austria (AT). Colors shown in the right panel correspond to the source country, and country codes shown are from Eurostat (34). 

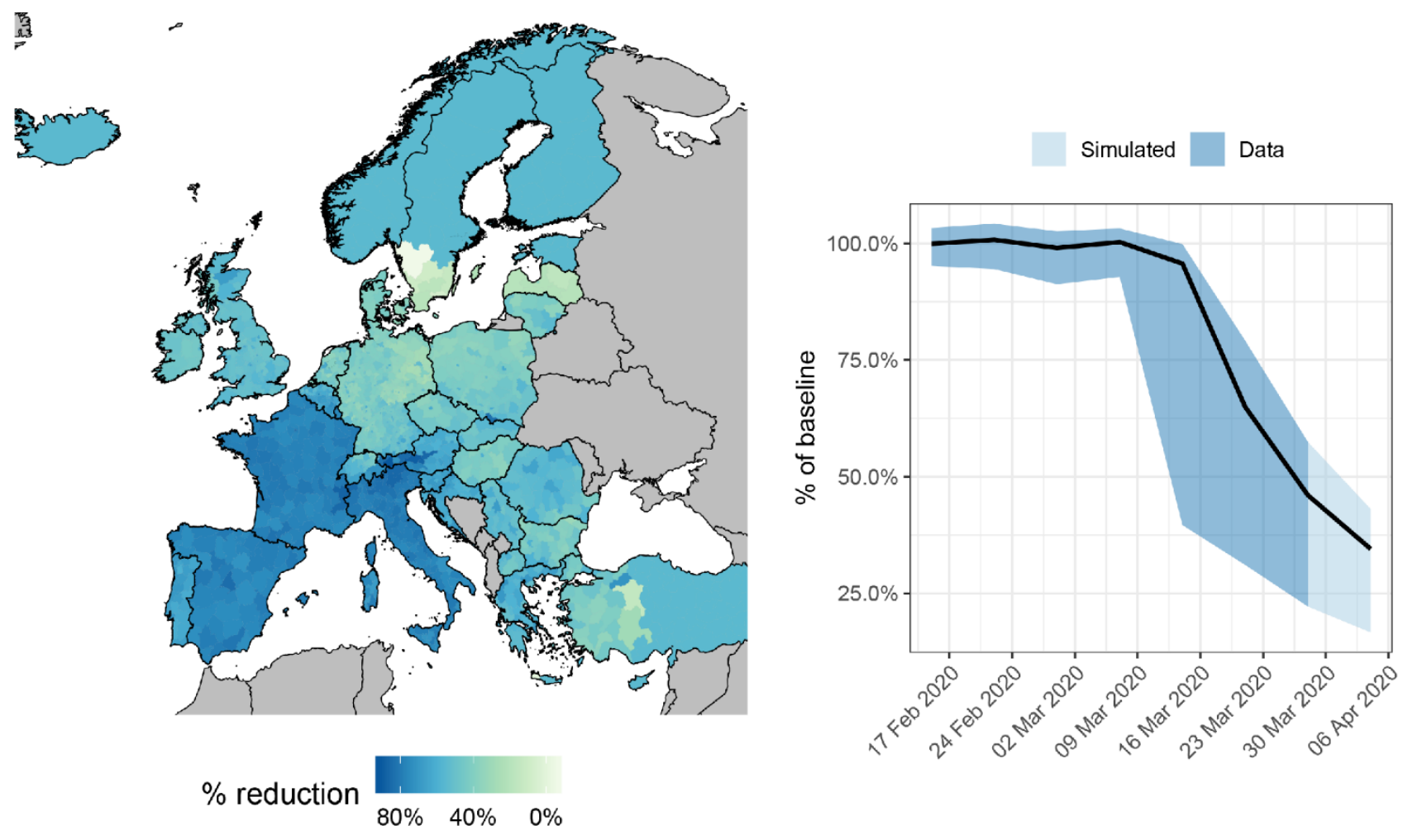

Fig. 2. Reduction in mobility observed in NUTS3 areas from 11 Feb to $6 \mathrm{Apr} 2020$. (Left) Reduction in mobility observed in each NUTS3 administrative unit across Europe for the week of 21-28 Mar 2020 compared to Jan-Feb 2020. Movement data were not available for countries in grey. (Right) Weekly average change in mobility across all NUTS3 areas. Dark blue shows reductions observed in the Google COVID-19 dataset, light blue shows extrapolation of reductions by one week. Black line shows mean change compared to baseline. When implementing NPIs in various NUTS3 areas, we used the movement reduction estimates for the end of this period, 6 Apr 2020. 


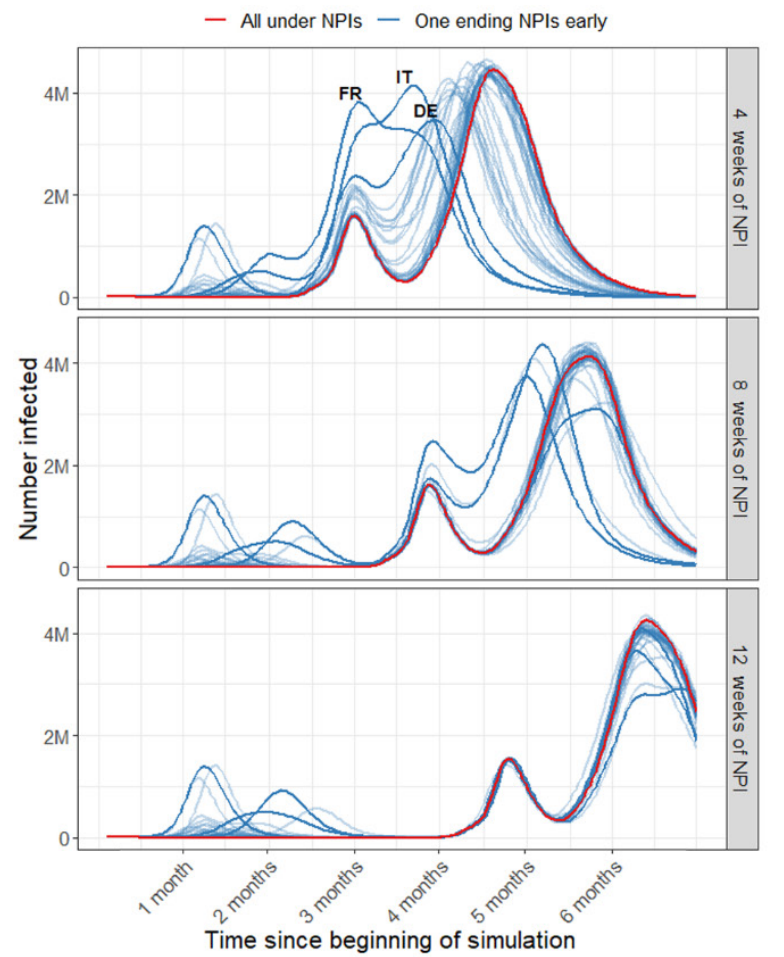

Earlier resurgence Europe-wide (in days) $\begin{array}{lll}0 & 15 & 30\end{array}$

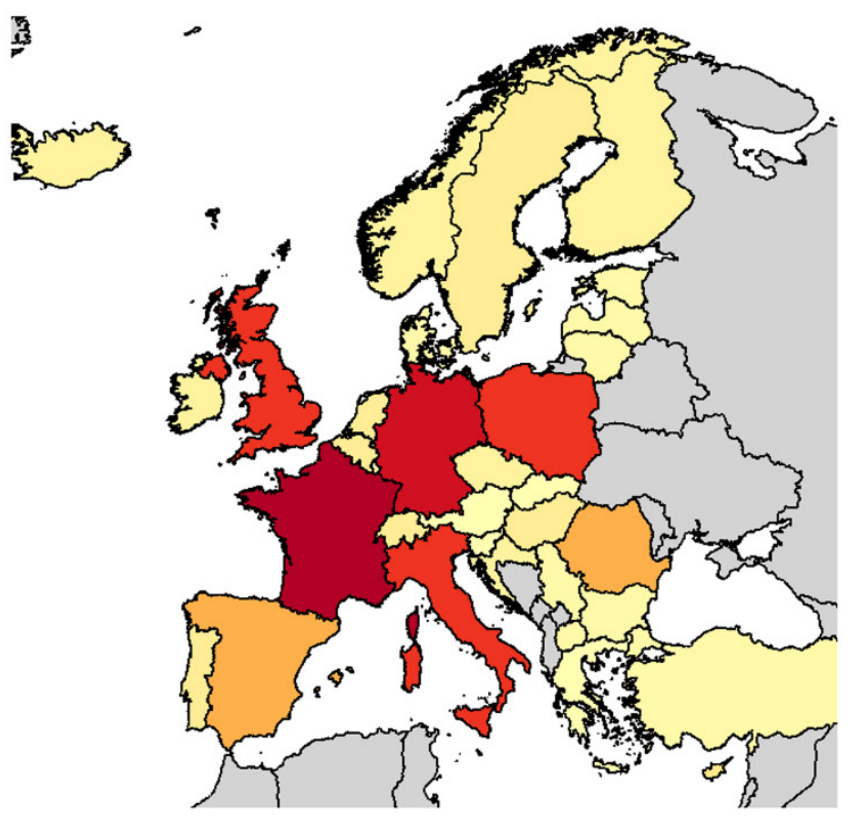

Fig. 3. Epidemic spread if all countries but one maintain existing NPIs. When lifting NPIs early, countries reverted to baseline mobility on 15 April. (Left) Epidemic curves, with varying numbers of weeks that NPIs are implemented. Curves indicate numbers of active cases at any given time, rather than numbers of new cases per day. Red lines indicate epidemic curves where all countries maintain NPIs for the denoted number of weeks. Blue lines indicate epidemic curves if one country ends intervention policies early (each line represents one randomly chosen country that ends its policies early); France, Germany, and Italy are highlighted. (Right) For the 4 weeks of NPI scenario, the number of days earlier that an uncontrolled second epidemic occurs continent-wide if each country ends NPIs early, measured as the time to $25 \%$ of the population of Europe having had COVID-19. Movement data were not available for countries in grey. 


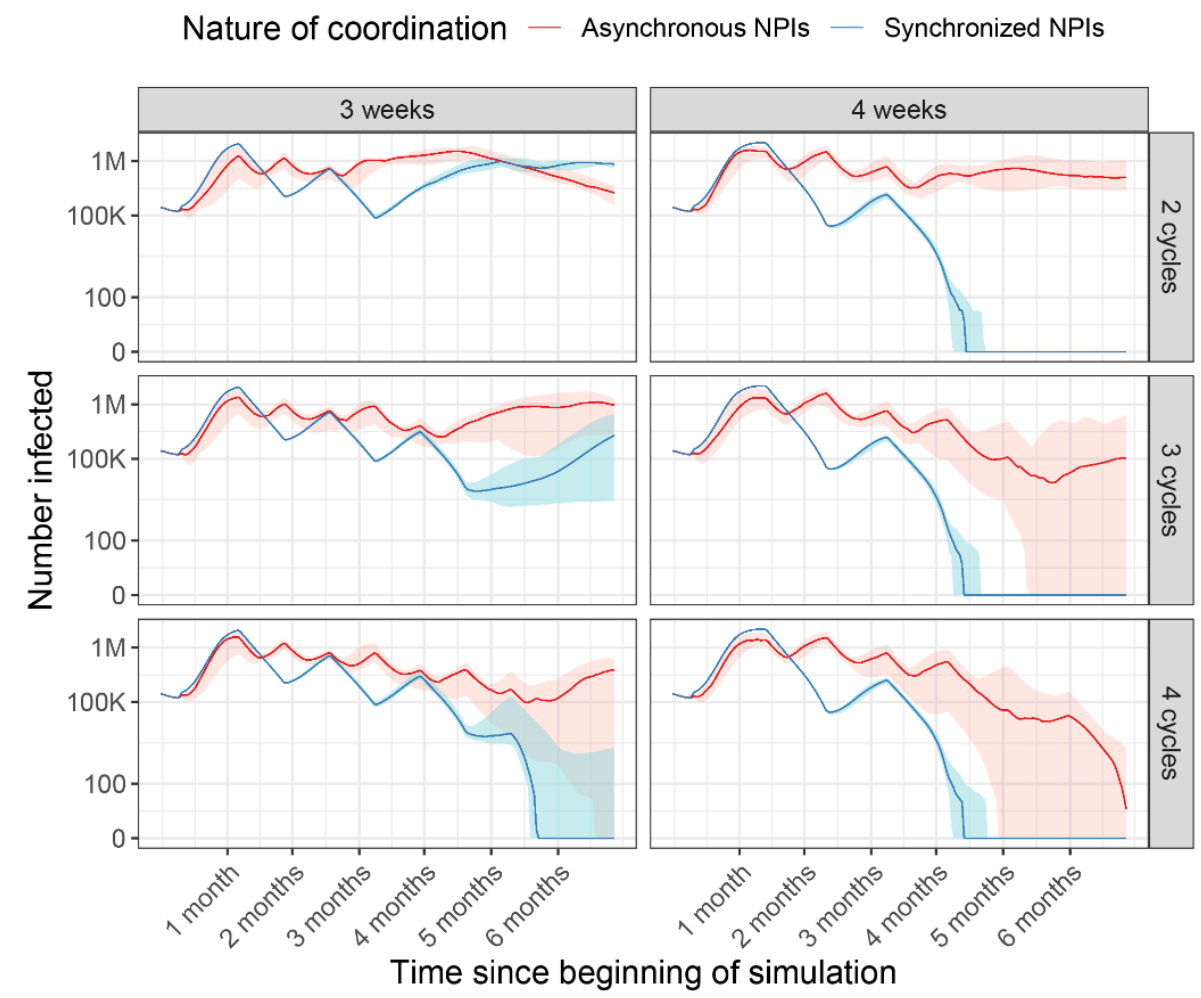

Fig. 4. Cases over time, when NPIs are synchronized and unsynchronized across all European countries. Rows vary the number of on-off cycles that occur, and columns indicate the number of on-off cycles implemented. For example, 4 weeks with 2 cycles (top right) indicates we simulated two cycles of 4 weeks on lockdown, 4 weeks off lockdown for each country. Red: Cases when European countries do not synchronize NPI timing. Blue: Cases when European countries are all synchronized in NPI timing. Shaded areas indicate intervals in which 95\% of simulations fell within, over 200 simulations. 